\title{
Early identification of familial hypercholesterolaemia in general practice using patient-specific reminders: focus group with General Practitioners
}

\author{
Jennifer Tranter, Joe Kai ${ }^{*}$, Nadeem Qureshi \\ From Health Services Research: Evidence-based practice \\ London, UK. 1-3 July 2014
}

\section{Background}

Familial Hypercholesterolaemia (FHc) is a common inherited disorder, leading to raised serum cholesterol evident from the first year of life. One in 500 people are affected by the minor heterozygote form of this condition, of which an estimated $85 \%$ are unidentified. National English guidelines, produced by NICE, highlight the importance of cholesterol screening and FHc identification in prevention of coronary heart disease $[1,2]$. However, evidence based approaches to support guideline implementation are under developed. The incorporation of computerised patient specific reminders is thought to have clinical utility in the identification of patients with genetic conditions but as yet not evaluated in primary care for FHc.

\section{Materials and methods}

In order to develop computer based prompts are acceptable to general practitioners a focus group was held for general practitioners from the Inner City Derby area, of which the outcome would inform the design of a future feasibility study to assess the use and impact of such reminders for FHc in clinical practice.

\section{Results}

General practitioners were in favour of specific patient reminders for early identification of individuals at risk of FHc. Emerging themes included brevity, clear and succinct statements, explicit actions that linked specific screening tests to specific diseases and the need to tailor different GP clinical systems.

\section{Conclusions}

Clinicians need to be alert to the prevalence of FHc in clinical practice. Based on results from both the focus group and preliminary work, PSR's were considered to be an overall effective method of early identification of FHc in general practice and therefore considered relevant in pre-trial work to inform substantive research in the early identification of FHc in primary care.

Published: 7 July 2014

\section{References}

1. Demott K, Nherera L, Humphries SE, Minhas R, Shaw EJ, Qureshi N, et al: Clinical Guidelines and Evidence Review for Familial Hypercholesterolaemia: the identification and management of adults and children with familial hypercholesterolaemia. London: National Collaborating Centre for Primary Care and Royal College of General Practitioners; 2008

2. Qureshi N, Humphries SE, Seed M, Rowlands P, Minhas R: Identification and management of familial hypercholesterolaemia: what does it mean to primary care? Brit J Gen Pract 2009, 59:773-776.

doi:10.1186/1472-6963-14-S2-P97

Cite this article as: Tranter et al:: Early identification of familial hypercholesterolaemia in general practice using patient-specific reminders: focus group with General Practitioners. BMC Health Services Research 201414 (Suppl 2):P97. 